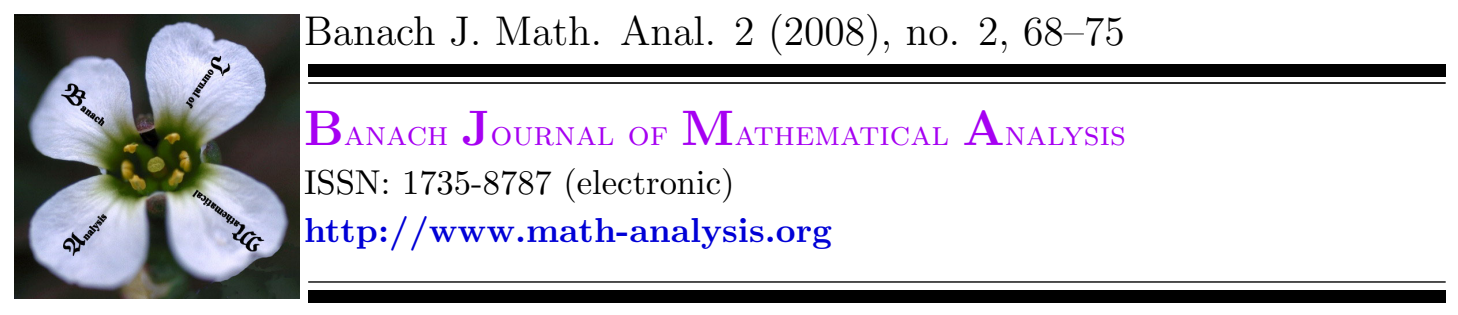

\title{
AN EIGENVALUE PROBLEM WITH MIXED BOUNDARY CONDITIONS AND TRACE THEOREMS
}

\author{
CATHERINE BANDLE ${ }^{1}$ \\ This paper is dedicated to Professor J. E. Pecaric \\ Submitted by P. K. Sahoo
}

\begin{abstract}
An eigenvalue problem is considered where the eigenvalue appears in the domain and on the boundary. This eigenvalue problem has a spectrum of discrete positive and negative eigenvalues. The smallest positive and the largest negative eigenvalue $\lambda_{ \pm 1}$ can be characterized by a variational principle. We are mainly interested in obtaining non trivial upper bounds for $\lambda_{-1}$. We prove some domain monotonicity for certain special shapes using a kind of maximum principle derived by C. Bandle, J.v. Bellow and W. Reichel in [J. Eur. Math. Soc., 10 (2007), 73-104]. We then apply these bounds to the trace inequality.
\end{abstract}

\section{INTRODUCTION AND PRELIMINARIES}

Let $D \subset \mathbb{R}^{N}$ be a bounded domain with a Lipschitz boundary and denote by $n$ its outer normal. The eigenvalue problem we are interested in is

$$
\triangle \varphi+\lambda \varphi=0 \quad \text { in } \quad D, \quad \frac{\partial \varphi}{\partial n}=\lambda \sigma \varphi \quad \text { on } \quad \partial D .
$$

Here $\sigma$ is a real number. Notice that $\lambda_{0}=0$ and $\varphi_{0}=$ const. is always a solution. It was proved in [1], cf. also [5] that problem (1.1) has a discrete spectrum

Date: Received: 21 April 2008; Accepted 20 May 2008.

2000 Mathematics Subject Classification. Primary 35P15, 47A75 ; Secondary 49R50, 51M16.

Key words and phrases. Estimates of eigenvalues, trace inequality, comparison theorems for eigenvalues. 
consisting of non negative eigenvalues

$$
\lambda_{0}=0<\lambda_{1}<\lambda_{2} \leq \lambda_{3} \leq \ldots \quad \text { if } \quad \sigma \geq 0
$$

and of positive and negative eigenvalues if $\sigma$ is negative. Taking into account their multiplicity they can be ordered as follows:

$$
\ldots \leq \lambda_{-n} \leq \ldots \leq \lambda_{-2} \leq \lambda_{-1}<0=\lambda_{0}<\lambda_{1} \leq \lambda_{2} \leq \ldots \leq \lambda_{n} \leq \ldots
$$

Except for $N=1$ both the positive and the negative parts of the spectrum contains infinitely many eigenvalues tending to $\pm \infty$. As for the classical eigenvalue problems the eigenvalues and eigenfunctions can be obtained by a minimum maximum principle [1. We shall describe it for $\lambda_{ \pm 1}$ and $\sigma<0$ which will be the main topic of this paper. For this purpose we introduce the following notation. For $u, v \in W^{1,2}(D)$ set

$$
\begin{array}{r}
a(u, v):=\int_{D} u v d x+\sigma \int_{\partial D} u v d s \\
<u, v>:=\int_{D}(\nabla u, \nabla v) d x
\end{array}
$$

The number

$$
\sigma_{0}(D):=-\frac{|D|}{|\partial D|}
$$

will play an essential role in our considerations. It turns out cf. [3] that

$$
\begin{array}{r}
\frac{1}{\lambda_{1}(D)}=\sup _{\mathcal{K}} a(v, v), \quad \text { if } \quad \sigma<\sigma_{0}<0, \\
\frac{1}{\lambda_{-1}(D)}=\inf _{\mathcal{K}} a(v, v) \quad \text { if } \quad \sigma_{0}<\sigma<0, \\
\mathcal{K}:=\left\{v \in W^{1,2}(D),<v, v>=1\right\} .
\end{array}
$$

From here it follows [1] that

(i) If $\sigma<\sigma_{0}$ then $\varphi_{1}$ is of constant sign and $\lambda_{1}$ is simple, whereas $\varphi_{-1}$ changes sign.

(ii) If $0>\sigma>\sigma_{0}$ then $\varphi_{-1}$ is of constant sign and $\lambda_{-1}$ is simple, whereas $\varphi_{1}$ changes sign.

(iii) If $\sigma=\sigma_{0}$ both $\varphi_{1}$ and $\varphi_{-1}$ change sign.

From 1.2 we get trace inequalities of the type

$$
\begin{gathered}
|\sigma| \oint_{\partial D} v^{2} d s \geq \int_{D} v^{2} d x-\frac{1}{\lambda_{1}(D)} \int_{D}|\nabla v|^{2} d x \quad \text { if } \quad \sigma<\sigma_{0}<0 \\
|\sigma| \oint_{\partial D} v^{2} d s \leq \int_{D} v^{2} d x+\frac{1}{\left|\lambda_{-1}(D)\right|} \int_{D}|\nabla v|^{2} d x \quad \text { if } \quad \sigma_{0}<\sigma<0 .
\end{gathered}
$$

In order to exhibit these inequalities we need a lower bound for $\lambda_{1}(D)$ and an upper bound for $\lambda_{-1}(D)$. Such bounds were given in [3] for $\lambda_{1}(D)$. In particular it was shown that among all domains of fixed volume $\lambda_{1}$ is smallest for the ball. 
Notice that unlike the case with Dirichlet boundary conditions the eigenvalues are in general not monotone with respect to the domain. An exception is when $D$ is contained in a ball $B, D \subset B$. Then [3], $\lambda_{1}(D) \geq \lambda_{1}(B)$ and $\lambda_{-1}(D) \geq \lambda_{-1}(B)$.

In this note we give some estimates from above for $\lambda_{-1}$ and compare the corresponding trace inequality (1.3) with a result of Horgan [4].

\section{MAin RESUlts}

Let us start with two examples.

2.1. Ball. Consider the the ball $B_{R}$ centered at the origin and of radius $R$ and let $(r, \theta), r \in[0, R], \theta \in \mathbb{S}^{N-1}$ be its polar coordinates. The critical value is

$$
\sigma_{0}=-\frac{R}{N}
$$

Separation of variables $\varphi(x)=w(r) \alpha(\theta)$ yields

$$
w^{\prime \prime}+\frac{N-1}{r} w^{\prime}+\left(\lambda-\frac{\nu}{r^{2}}\right) w=0, \quad \Delta_{\theta} \alpha+\nu \alpha=0,
$$

where $\Delta_{\theta}$ is the Laplace-Beltrami operator on $\mathbb{S}^{N-1}$ with eigenfunction $\alpha$ and eigenvalue $\nu$. Hence $\alpha$ must be a spherical harmonic and $\nu=\nu_{k}=k(k+N-2)$, $k=0,1,2, \ldots$. We are interested only in the case where the eigenfunctions do not change sign. Therefore $\nu=0$ and $\alpha=1$. The equation for $w$ then becomes

$$
\begin{aligned}
w^{\prime \prime}+\frac{N-1}{r} w^{\prime}+\lambda w & =0 \text { in }(0, R), \\
w^{\prime}(R) & =\sigma \lambda w(R) .
\end{aligned}
$$

By the usual transformation $z(r)=r^{\frac{N-2}{2}} w(r)$ one finds

$$
\begin{gathered}
z^{\prime \prime}+\frac{z^{\prime}}{r}+\left(\lambda-\frac{((N-2) / 2)^{2}}{r^{2}}\right) z=0 \text { in }(0, R), \\
z^{\prime}(R)=\left(\sigma \lambda+\frac{N-2}{2 R}\right) z(R) .
\end{gathered}
$$

Solutions are of the form

$$
z(r)= \begin{cases}J_{(N-2) / 2}(\sqrt{\lambda} r) & \text { if } \lambda>0 \\ I_{(N-2) / 2}(\sqrt{-\lambda} r) & \text { if } \lambda<0 \\ r^{(N-2) / 2} & \text { if } \lambda=0\end{cases}
$$

where $J_{\nu}$ is the regular Bessel function of index $\nu$ and $I_{\nu}$ is the regular modified Bessel function of index $\nu$. The eigenvalues $\lambda_{ \pm 1}$ are determined by the equations

$$
\begin{aligned}
\frac{J_{(N-2) / 2}^{\prime}(\sqrt{\lambda} R)}{J_{(N-2) / 2}(\sqrt{\lambda} R)} & =\frac{\sigma \lambda+\frac{N-2}{2 R} \text { if } \lambda>0,}{\sqrt{\lambda}} \\
\frac{I_{(N-2) / 2}^{\prime}(\sqrt{-\lambda} R)}{I_{(N-2) / 2}(\sqrt{-\lambda} R)} & =\frac{\sigma \lambda+\frac{N-2}{2 R}}{\sqrt{-\lambda}} \text { if } \lambda<0 .
\end{aligned}
$$

If follows from the remark at the end of the Introduction that $\lambda_{ \pm 1}$ is a decreasing function of $R$. 
2.2. $\mathbf{N}$-dimensional rectangle. Consider the rectangle $\mathbf{R}:=\left\{x \in \mathbb{R}^{N}:\left|x_{i}\right|<\right.$ $\left.a_{i}\right\}, i=1,2 \ldots N$. Separation of variables yields for the eigenfunctions corresponding to $\lambda_{ \pm 1}$

$$
\begin{aligned}
\varphi_{1} & =\prod_{i=1}^{N} \cos \left(\sqrt{\omega_{1}} x_{i}\right), \quad \lambda_{1}=\sum_{i=1}^{N} \omega_{i} \\
\varphi_{-1} & =\prod_{i=1}^{N} \cosh \left(\sqrt{\nu_{1}} x_{i}, \lambda_{-1}=-\sum_{i=1}^{N} \nu_{i}\right.
\end{aligned}
$$

where

$$
-\sqrt{\omega_{i}} \tan \left(\sqrt{\omega_{i}} a_{i}\right)=\lambda_{1} \sigma, \quad \sqrt{\nu_{i}} \tanh \left(\sqrt{\nu_{i}} a_{i}\right)=\lambda_{-1} \sigma .
$$

For a cube $\mathcal{C}_{a}$ where $a_{i}=a$, we get $\omega_{i}=\omega$ and $\nu_{i}=\nu$ for all $i$ where

$$
-\tan (\sqrt{\omega} a)=N \sqrt{\omega} \sigma, \quad \tanh \sqrt{\nu} a=N \sqrt{\nu} \sigma .
$$

The critical value for $\mathbf{R}$ is

$$
\sigma_{0}=-\left(\sum_{j=1}^{N} \frac{1}{a_{j}}\right)^{-1} .
$$

It follows from the previous remark that $\omega_{i} \neq 0, i=1 \ldots N$ exist only if $\sigma<\sigma_{0}$ and $\nu_{i} \neq 0, i=1, \ldots, N$ exist only if $\sigma>\sigma_{0}$.

Monotonicity Let $a_{1} \leq a_{2} \leq \ldots a_{N}$. Since $x \rightarrow x \tan (a x)$ and $x \rightarrow \tanh (a x)$ are increasing functions of $a$ for fixed $x>0$, we deduce that $\omega_{1} \geq \omega_{2} \geq \cdots \geq \omega_{N}$ and $\nu_{1} \geq \nu_{2} \geq \cdots \geq \nu_{N}$.

Lemma 2.1. (i) If $\sigma<\sigma_{0}$ then

$$
\lambda_{1}\left(\mathcal{C}_{a_{N}}\right) \leq \lambda_{1}(\mathbf{R}) \leq \lambda_{1}\left(\mathcal{C}_{a_{1}}\right)
$$

(ii) Let $\sigma>\sigma_{0}$. Then

$$
\lambda_{-1}\left(\mathcal{C}_{a_{N}}\right) \leq \lambda_{-1}(\mathbf{R}) \leq \lambda_{-1}\left(\mathcal{C}_{a_{1}}\right)
$$

Proof. (i) The inequality $\omega_{1} \geq \omega_{i}$ implies

$$
\sqrt{\omega_{1}} \tan \left(\sqrt{\omega_{1}} a_{1}\right)=\lambda_{1}|\sigma| \leq N \omega_{1}|\sigma|
$$

Let $\omega^{*}$ be the solution

$$
\sqrt{\omega^{*}} \tan \left(\sqrt{\omega^{*}} a_{1}\right)=N \omega^{*}|\sigma| .
$$

Note that for positive $a$, the function $x \rightarrow \frac{\tan (x a)}{x}$ is increasing in $(0, \pi / 2 a)$. Hence $\omega_{1} \leq \omega^{*}$ and by $(2.1)$

$$
\sqrt{\omega^{*}} \tan \left(\sqrt{\omega^{*} a_{1}} \geq \sqrt{\omega_{1}} \tan \left(\sqrt{\omega_{1} a_{1}}=\lambda_{1}|\sigma| .\right.\right.
$$

The first assertion now follows from (2.2). The proof of the second assertion is similar with the inequality signs reversed.

(ii) The inequality $\nu_{1} \geq \nu_{i}$ implies

$$
\sqrt{\nu_{1}} \tanh \left(\sqrt{\nu_{1}} a_{1}\right)=\lambda_{-1}|\sigma| \leq N \nu_{1}|\sigma|
$$


Let $\nu^{*}$ be the solution

$$
\sqrt{\nu^{*}} \tanh \left(\sqrt{\nu^{*}} a_{1}\right)=N \nu^{*}|\sigma| .
$$

Note that for positive $a$, the function $x \rightarrow \frac{\tanh (x a)}{x}$ is decreasing in $\mathbb{R}^{+}$. Hence $\nu_{1} \geq \nu^{*}$ and by 2.1

$$
\sqrt{\nu^{*}} \tanh \left(\sqrt{\nu^{*} a_{1}} \leq \sqrt{\nu_{1}} \tanh \left(\sqrt{\nu_{1} a_{1}}=\lambda_{-1}|\sigma| .\right.\right.
$$

The first conclusion follows from $(2.2)$. The second assertion is obtained by the same argument.

\subsection{General domains.}

2.3.1. Scaling. Let $\alpha>0$ be an arbitrary fixed number. Put $y=\alpha x$. If $(\varphi, \lambda)$ is a solution of 1.1 then $\psi(y)=\varphi(y / \alpha)$ satisfies

$$
\triangle \psi+\frac{\lambda}{\alpha^{2}} \psi=0 \quad \text { in } \quad \alpha D, \quad \frac{\partial \psi}{\partial n}=\alpha^{-1} \lambda \sigma \psi \quad \text { on } \quad \partial(\alpha D) .
$$

Hence $\lambda \alpha^{-2}$ corresponds to the eigenvalue in $\alpha D$ with $\sigma$ replaced by $\alpha \sigma$. It was shown in [2] that for $\sigma \in \mathbb{R}^{-}$the functions $\sigma \rightarrow \sigma \lambda_{ \pm 1}(\sigma)$ are continuous and strictly increasing. Hence for $\alpha>1$

$$
\frac{\lambda_{ \pm 1}(D)}{\alpha} \geq \lambda_{ \pm 1}(\alpha D)
$$

From this inequality we obtain that for any domain $D$ and any $\alpha>1$ the following monotonicity result

$$
\lambda_{1}(D) \geq \lambda_{1}(\alpha D)
$$

Such a conclusion can not be drawn for $\lambda_{-1}$.

Open problem Does $\lambda_{-1}(D) \geq \lambda_{-1}(\alpha D)$ hold for all domains?

Remark 2.2. It is not difficult to see that the above inequality holds for the balls and the rectangles.

2.3.2. Positivity principle and upper bounds for $\lambda_{-1}$. Nontrivial upper bounds for $\lambda_{-1}$ can be obtained from the positivity principle derived in [2].

Lemma 2.3. Assume $\sigma \in\left(\sigma_{0}, 0\right)$. If there exists a positive solution $u$ of

$$
\triangle u+\lambda u \leq 0 \quad \text { in } \quad D, \quad \frac{\partial u}{\partial n} \geq \lambda \sigma u \quad \text { on } \quad \partial D
$$

then $\lambda \geq \lambda_{-1}$.

Theorem 2.4. Let $D$ be a star-shaped domain and $B_{R}$ the greatest ball inscribed in D. Suppose that $\left(\frac{x}{|x|}, n\right) \geq \alpha$ on $\partial D$ and that $\sigma^{\prime}:=\sigma \alpha^{-1} \geq \sigma_{0}\left(B_{R}\right)=-R / N$. Let $\lambda_{-1}^{\prime}\left(B_{R}\right)$ be the largest negative eigenvalue of 1.1 corresponding to $\sigma^{\prime}$. Then

$$
\lambda_{-1}(D) \leq \lambda_{-1}^{\prime}\left(B_{R}\right)
$$


Proof. Let $u$ be the eigenfunction of $B_{R}$ corresponding to $\lambda_{-1}^{\prime}\left(B_{R}\right)$ with $\sigma$ replaced by $\sigma^{\prime}:=\sigma / \alpha$. This eigenfunction can be extended to $r>R$ (cf. Section 2.1). From the monotonicity of $\lambda_{-1}^{\prime}\left(B_{R}\right)$ with respect to $R$ (cf. [3]) it follows that the function $r \rightarrow u^{\prime}(r) / u(r)$ is increasing. This can also be seen directly from the following simple argument. Consider the function $v=\frac{u^{\prime}(r)}{u(r)}$. It satisfies

$$
v^{\prime}+v^{2}+\frac{N-1}{r} v+\lambda_{-1}^{\prime}=0 \quad \text { in } \quad \mathbb{R}^{+}, \quad v(0)=0 .
$$

Near $r=0$ obviously $v$ increases. Suppose that $v$ is not monotone. Then there exists a point $r^{\prime}$ where $v$ assumes a local maximum. This is not possible because $v^{\prime \prime}\left(r^{\prime}\right)=\frac{N-1}{r^{\prime}} v\left(r^{\prime}\right)>0$. On $\partial D$ we have

$$
\frac{\partial u}{\partial n}=\left.u^{\prime}(r)\left(\frac{x}{|x|}, n\right)\right|_{\partial D} \geq\left.\lambda_{-1}^{\prime} \sigma^{\prime} \alpha u(r)\right|_{\partial D}=\left.\lambda_{-1}^{\prime} \sigma u(r)\right|_{\partial D} .
$$

The assertion now follows from Lemma 2.3.

A similar result can be obtained if we inscribe instead of a ball a rectangle $\mathbf{R}$ as in Section 2.2.

Theorem 2.5. Let $D$ be convex and $\mathbf{R} \subset D$. Assume that for $i=1, \ldots N$, $\operatorname{sign}\left(n_{i}\right)=\operatorname{sign}\left(x_{i}\right)$ on $\partial D$. (This can always be achieved in convex domains.) Let $\lambda_{-1}^{\prime \prime}$ be the first negative eigenfunction of $\mathbf{R}$ with $\sigma$ replaced by $\sigma^{\prime \prime}:=\sigma \beta^{-1}$, where $\beta=\min _{\partial D} \sum_{i=1}^{N}\left|n_{i}\right|$. Suppose that $\sigma^{\prime \prime}>\sigma_{0}(\mathbf{R})$. Then

$$
\lambda_{-1}(D) \leq \lambda_{-1}^{\prime \prime}(\mathbf{R}) \text {. }
$$

Proof. Let $\varphi$ be the positive eigenfunction corresponding to $\mathbf{R}$ (cf. Section 2.2). Then since $\mathbf{R}$ is contained in $D$ we have, using the same notation as in Section 2.2 .

$$
\left.\varphi^{-1} \frac{\partial \varphi}{\partial n}\right|_{\partial D}=\sum_{j=0}^{N} n_{j} \frac{x_{j}}{\left|x_{j}\right|}\left|\nu_{j}\right| \tanh \left(\sqrt{\left|\nu_{j}\right|} x_{j}\right) \geq \beta \sigma^{\prime \prime} \lambda_{1}^{\prime \prime}=\sigma \lambda_{-1}^{\prime \prime} .
$$

The conclusion follows now from Lemma 2.3.

Remark 2.6. From the last theorem we see immediately that if $\mathbf{R}^{\prime}$ and $\mathbf{R}$ are two rectangles such that $\mathbf{R}^{\prime} \subset \mathbf{R}$ then $\lambda_{-1}(\mathbf{R}) \leq \lambda_{-1}\left(\mathbf{R}^{\prime}\right)$.

Open problem Is it possible to find other domains beside of balls and rectangles for which inclusion results as in Theorems 2.4 and 2.5 hold?

\section{Trace Inequalities}

It is well-known that for Lipschitz domains a function $v \in W^{1,2}(D)$ has a trace which satisfies the following inequality: for any given $\epsilon>0$ there exists a positive number $c(\epsilon)$ independent of $v$ such that

$$
\oint_{\partial D} v^{2} d s \leq \epsilon \int_{D}|\nabla v|^{2} d x+c(\epsilon) \int_{D} v^{2} d x
$$


The smallest such constant will be denoted by $c^{*}(\epsilon)$. For star-shaped domains Horgan [4] has shown that

$$
c^{*}(\epsilon) \leq \frac{N}{A}+\frac{T^{2}}{A^{2} \epsilon} \quad \text { where } A=\min _{\partial D}(x, n) \text { and } \quad T=\max _{\partial D}|x| .
$$

Equations (3.1) and $(3.2)$ can be used to estimate $\lambda_{-1}(D)$ from above provided $|\sigma|<A / N$. Namely, choosing $\epsilon=\frac{T^{2}}{A^{2}\left(|\sigma|^{-1}-N / A\right)}$, we obtain

$$
\frac{1}{\lambda_{-1}(D)}=\int_{D} \varphi_{-1}^{2} d x+\sigma \oint_{\partial D} \varphi_{-1}^{2} d s \geq \frac{\sigma T^{2}}{A^{2}\left(|\sigma|^{-1}-N / A\right)} .
$$

Observe that this is a rather crude bound because the inequality (3.1) is not optimal in contrast to 1.3 which is attained for $v=\varphi_{-1}$. Another disadvantage is that this estimate does not yield an upper bound if $-\sigma_{0}<\sigma<-A / N$. From (1.3) we get we obtain

$$
\oint_{\partial D} v^{2} d s \leq \frac{1}{\left|\sigma \lambda_{-1}(D)\right|} \int_{D}|\nabla v|^{2} d x+|\sigma|^{-1} \int_{D} v^{2} d x \quad \text { if } \quad \sigma_{0}<\sigma<0 .
$$

It turns out [2] that

$$
\sigma \lambda_{-1}(D ; \sigma) \nearrow, \quad \lim _{\sigma \rightarrow \sigma_{0}^{+}} \sigma \lambda_{-1}(D ; \sigma)=0 \quad \lim _{\sigma \rightarrow 0^{-}} \sigma \lambda_{-1}(D ; \sigma)=\infty .
$$

Consequently for any given $\epsilon>0$ we can find $\sigma_{0}<\sigma_{\epsilon}<0$ such that $\sigma_{\epsilon} \lambda_{-1}\left(D ; \sigma_{\epsilon}\right)=\epsilon$.

Corollary 3.1. Let $D$ be an arbitrary (not necessarily star-shaped) Lipschitz domain. Let $\sigma_{\epsilon}$ be the unique solution of $\sigma \lambda_{-1}(D)=\epsilon^{-1}$. Then the optimal constant $c^{*}(\epsilon)$ in (3.1) is $\left|\sigma_{\epsilon}^{-1}\right|$. The equality sign holds for the corresponding eigenfunction $\varphi_{-1}$.

Under the conditions of Theorem 2.4 we can replace $\lambda_{-1}(D)$ by the upper bound $\lambda_{-1}^{\prime}\left(B_{R}\right)$. If $\tilde{\sigma}_{\epsilon}$ is the solution of $\sigma \lambda_{-1}^{\prime}\left(B_{R}\right)=\epsilon^{-1}$ then the optimal constant satisfies

$$
c^{*}(\epsilon) \leq \frac{1}{\left|\tilde{\sigma}_{\epsilon}\right|}
$$

\section{REFERENCES}

1. C. Bandle, J.v. Below and W. Reichel, Parabolic problems with dynamical boundary conditions: eigenvalue expansions and blow up, Rend. Lincei Mat. Appl., 17 (2006), 35-67.

2. C. Bandle, J.v. Below and W. Reichel, Positivity and anti-maximum principles for elliptic operators with mixed boundary conditions, J. Eur. Math. Soc., 10 (2007), 73-104.

3. C. Bandle, A Rayleigh-Faber-Krahn inequality and some monotonicity properties for eigenvalue problems with mixed boundary conditions, to appear in Proceedings of the Conference on Inequalities and Applications '07.

4. C.O. Horgan, Eigenvalue estimates and the trace theorem, J. Math. Anal. Appl., 69 (1979), 231-242.

5. J. L. Vazquez and E. Vitillaro, Heat equation with dynamical boundary conditions of locally reactive type, Semigroup Forum, 74 (2007), no. 1, 1-40. 
${ }^{1}$ Department of Mathematics, University of Basel, Rheinsprung 21, CH-4051 Basel, SWitzerland.

E-mail address: catherine.bandle@unibas.ch 